\title{
VATS Left Pneumonectomy with Pericardial defect: Clinical Case Examples
}

\author{
Yuang Mao, Yiping Wei*, Jianyong Zhang, Wenxiong Zhang, Yiming Wang and Dongliang Yu \\ Department of Cardiothoracic Surgery, the Second Affiliated Hospital of Nanchang University, Nanchang, People's Republic of China
}

Submission: July 04, 2019; Published: August 21, 2019

"Correspondence Author: Yiping Wei, Department of Cardiothoracic Surgery, the Second Affiliated Hospital of Nanchang University, Nanchang, People's Republic of China, Fuzhou Road 96 Rome, China

\begin{abstract}
We report the case of a 54-year-old asymptomatic male, who presented to us with Stage a B squamous cell carcinoma of the lung. We performed video-assisted thoracoscopic surgery (VATS) left pneumonectomy. During VATS, a complete left-sided absence of the pericardium was unexpectedly discovered, but we did not convert to a thoracotomy. We reviewed the preoperative chest CT, which showed the left heart was abnormal to the left, but the left pericardial defect was not obvious. If the patient is asymptomatic, complete pericardial defects usually do not endanger the patient's life, even in left pneumonectomy, there is no need for surgical repair. VATS left pneumonectomy with pericardial defect may lead to arrhythmia and a decrease in blood pressure, but as long as attention is paid to protecting the outer membrane of the heart, the coronary artery, and the vein of the heart during separation of adhesion lit is not necessary to convert to a thoracotomy. In conclusion, discovery of a pericardial defect during a VATS left pneumonectomy is not an indication of thoracotomy.
\end{abstract}

Keywords: VATS; Left Pneumonectomy; Pericardial defect

\section{Introduction}

Congenital pericardial defects are a rare developmental defect, affecting the left side more commonly [1]. The diagnosis of a partial or total congenital pericardial defect is seldom established before surgery, although this malformation is usually easily recognized if its existence is considered. According to the literature, a pericardial defect is often not handled. there are no related reports on how to treat the left total pneumonectomy with a pericardial defect under VATS, therefore without information or experience on how to handle. Although with no package protection during surgery, intraoperative surgical instruments are prone to interference of cardiac ventricular arrhythmia and hemodynamics of transient instability, we didn't perform a converted VATS procedure. The patient recovered well after the operation, and he was discharged on be hospitalized day 14. Provided there is control over interference to the heart, we believe that complete thoracoscopic pericardial absence of left pneumonectomy is feasible, and thoracotomy is not always required. Case Presentation and Surgical Technique

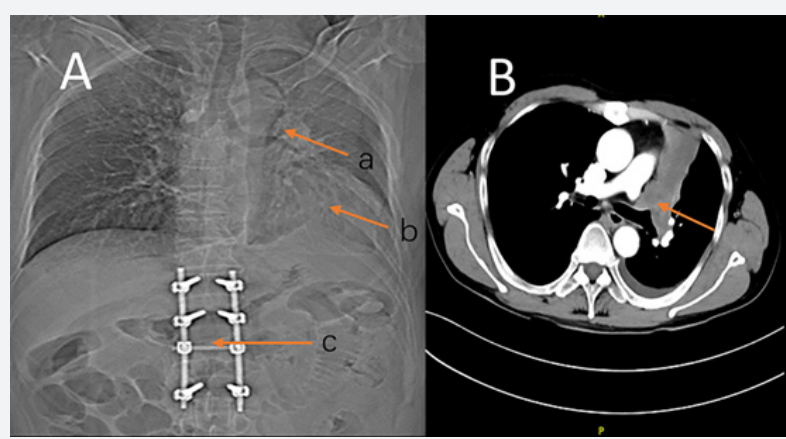

Figure 1: Enhanced computed tomographic scan.

A. Chest radiograph of a mass shadow in the left upper lung field adjacent to the mediastinum, left ventricular deviation. There is a steel plate on the spine 2013;

B. Left upper lobe bronchus near the hilum to see a lobulated mass shadow, about the size of $4 \mathrm{~cm} \times 2.5 \mathrm{~cm}$, the boundary is not clear, uneven density, with patchy vision $\square$ pleural effusion in the left thoracic cavity, but no tumor metastasis was found in the examination of hydrothorax. 


\section{Cancer Therapy \& Oncology International Journal}

A 54-year-old man with chest CT abnormality was referred to our hospital (Figure 1). The patient had no history of hypertension and diabetes. He underwent lumbar surgery for trauma in 2013. The patient was generally in good condition at admission. His physical examination revealed blood pressure 103/69mmHg, enhanced left lung fibrillation, and weaker left lung breathing. Heart auscultation area was normal. The abdomen was soft with no tenderness and rebound tenderness. Computed tomography (CT) of the chest demonstrated a left upper lobe bronchus near the hilum with a lobulated mass shadow, which was about $4 \mathrm{~cm} \times 2.5 \mathrm{~cm}$. The boundary was not clear, with uneven density. There was a pleural effusion in the left thoracic cavity, but no tumor metastasis was found in the examination of hydrothorax. Electronic bronchoscopy mucosa biopsy confirmed squamous-cell carcinoma. Blood biochemistry, electrocardiogram and preoperative examination showed no abnormalities. Pulmonary function test and blood gas analysis was normal.

The patient subsequently underwent VATS left pneumonectomy, who was first underwent intravenous anesthesia and a double-lumen endotracheal tube is used for single-lung ventilation. The process of VATS left pneumonectomy is as follow. Three trocars were used. A $3 \mathrm{~cm}$ working trocar in the 4rd intercostal space at the anterior axillary line. A 1.6$\mathrm{cm}$ thoracoscope was used at the mid-axillary line at the 8th intercostal space. A $1.5 \mathrm{~cm}$ assistant's trocar was placed in the 8th intercostal space in the scapular line. The process of the treatment of the lower pulmonary ligament revealed an obvious left mediastinal shift. When we isolated the left inferior vein, we found that there was frequent hypotension and arrhythmia in the compression of the left pericardium. Careful observation showed the absence of pericardium We were very careful when separating the heart from the lungs to avoid damaging the heart and heart vessels. We avoided excessive stimulation of the heart. Then a systematic complete lymph node dissection was done upon completion of the procedure. During the subcarinal lymph node dissection, we communicated to the anesthesiologists to observe closely because we suspend operative interference if the heart rate was more than 120 or less than 60 times, or when the blood pressure was lower than $85 / 55 \mathrm{mmHg}$. It can be recovered within a few minutes. The operation was completed under VATS, and there was no transition to the thoracotomy.

The patient's postoperative course was uneventful. There was no postoperative arrhythmia or hypotension. Postoperative pathology revealed moderately differentiated squamous cell carcinoma of the left lung, T2N1M0, stage ?B, with three lymph node metastases. Antibiotic treatment, expectorant, and other supportive treatment were given after operation. The patient was discharged 9 days since the resection. The thoracic CT and the electrocardiogram are reviewed, we find that the preoperative electrocardiogram was normal, a transthoracic echocardiogram was also implemented after the surgery, with non-specific discovering. The postoperative chest CT show the heart rotated clockwise around the venae cavae (Figure 2); However, the patient's postoperative course was uneventful, who did not go through adjuvant chemotherapy and has been symptom free for 2 months.

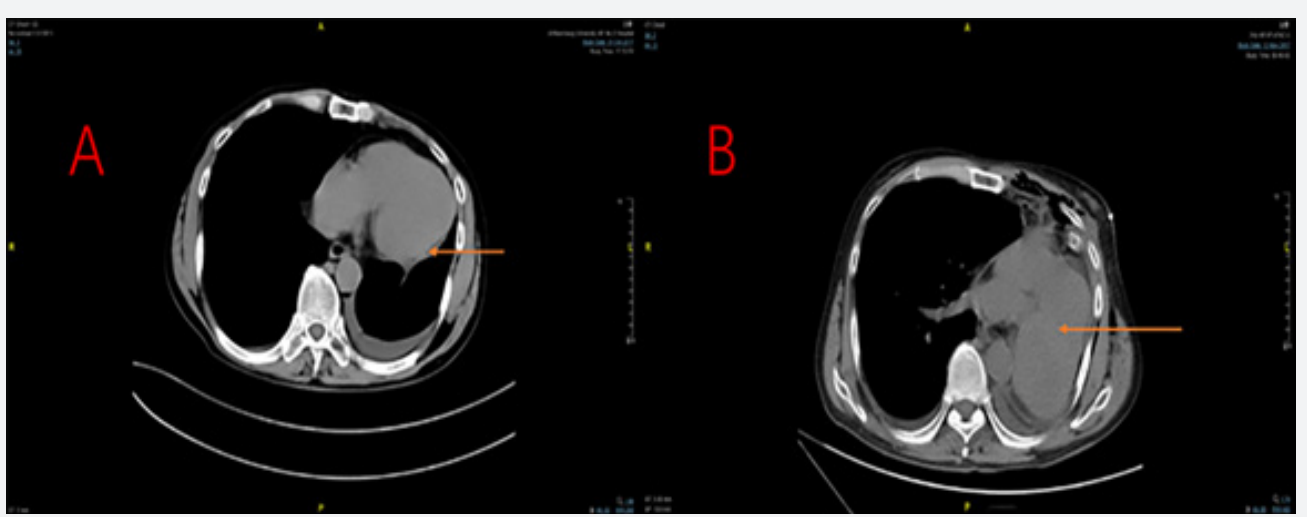

Figure 2: Preoperative enhanced computed tomographic scan versus enhanced postoperative computed tomographic scan.

A. Preoperative CT of the heart extending into the left pleural cavity, with no pericardial defect shown;

B. Post-operative CT of the heart rotated clockwise around the venae cavae.

\section{Discussion}

Patients with congenital pericardial defects is an uncommon condition, which was first described by Realdus Columbus in 1559 [2]. There are 6 categories of pericardial defects include complete left-sided absence, partial left-sided absence, complete right-sided absence, partial right-sided absence, total absence, and diaphragmatic defects [3]. Congenital pericardial absence is as a result of premature atrophy of the left common cardiac vein, which can lead to pleuropericardium dysplasia because of the insufficient blood supply [4,5]. Bilateral complete or right-sided pericardial defect is very uncommon, and a partial left-sided pericardial absence is more rare complete left-sided pericardial absence the lack of a pericardium is often found in surgical procedures such as cardiac surgery and mediastinal cysts. Yoshihisa et al. [6] suggested that a case of the lung cancer with the absence of the pericardium in thoracotomy. There has 


\section{Cancer Therapy \& Oncology International Journal}

been report of congenital pericardial defects be related to many congenital anomalies, however, a lung cancer patient underwent VATS left Pneumonectomy with pericardial defect has not been reported.

The operative statistics about the proportion of pericardial defects is $1-4 / 1000$. The proportion of morbidity in females to males is $1: 3$ in about $30 \%$ of reported cases [7]. Pericardial defect is usually diagnosed as an accidental finding, used for other indications during cardiovascular or thoracic diagnostic work, during postmortem autopsy or cardiothoracic surgery [8]. This patient's congenital pericardial defect was also found during the operation, but we did not have the time to find the pericardial absence due to the irritability of the heart. We found the pericardium by careful observation.

Although the incidence of pericardial defects is very rare, it can have disastrous consequences if not considered during the operation. The absence of pericardium usually has no obvious symptoms, but the fatal complications is associated with the congenital pericardial defect, for example, sudden death myocardial ischemia, myocardial strangulation, which is important for clinical detection. Symptoms depend on the size of the pericardial defect and vary between patients [7]. In our patient's case, although the heart was rotated toward the left pleural cavity, the postoperative course was uneventful, and the patient has been symptom free for 2 months since operation. Very small defects and complete pericardial defect with no fatal potential require no intervention [9]. For asymptomatic patients, it is not recommended to repair the pericardium even if the pericardium is found to be absent 6 . In this case, because the patient has no symptoms, we decided not to repair the pericardium.

While diagnosis of pericardial defects can be made by symptoms, ECG, X-ray, CT, and MRI, their sensitivity and specificity are not ideal. The absence of pericardium is most often incidentally discovered during surgery [3]. The bradycardia and right bundle branch block are typically appeared in ECG. R-wave progression secondary to left shift of the anterior thoracic transitional zone is common, and a prominent P-wave in the middle thoracic lead indicates right atrial overload [5]. Chest $\mathrm{X}$-rays typically show the outline of the heart, which shifts to the left, and the edge of the right heart disappears due to the superposition of the spine [7]. On CT, the entire pericardium or part of it does not exist. Other findings include axial left and backward displacement of the heart, alterations in the axis of the main pulmonary artery, enlargement of the atrial appendage or pulmonary artery segment, and separation of the aorta from the main pulmonary artery due to pulmonary interposition. The gated cardiac MRI with T1-weighted morphological sequences have good soft tissue contrast resolution that is able to delineate the fine, low-signal intensity of the normal pericardium [9].

However, this patient's lack of pericardium was only diagnosed under intraoperative conditions, in part by his history of trauma in 2013 with a large steel plate present on the spine and left closed drainage, with left pleurisy. Although the preoperative chest X-ray CT suggested that the mediastinum was more to the left than the average person, the imaging changes of the left chest did not directly associate with the absence of pericardium. Combined with intraoperative conditions, the above CT and X - ray are likely to suggest pericardial lesions.

\section{Conclusion}

In recent years, thoracoscopic surgery is becoming more and more popular. Compared with conventional thoracotomy, the advantages of VATS for lung cancer is obvious, such as reduced postoperative pain and intraoperative blood loss; surgical trauma; less operative time; fewer complications; less effect on pulmonary functions; and rapid recovery $[10,11]$. Survival rate is similar to that of conventional open surgery. Nonetheless, the operation time of the VATS group was longer than that of the thoracotomy group. This is because VATS patients need longer anastomosis than thoracotomy patients. In our patients, we suspend operative interference if the heart rate is more than 120 or less than 60 times, or when blood pressure drops lower than $85 / 55 \mathrm{mmHg}$. It is necessary to avoid excessive direct stimulation of the heart during the operation because of the unintentional package. In particular, the treatment of the inferior pulmonary veins, the inferior lung ligaments and the seventh groups of lymph nodes, prolong the operation time. Because the patient had no pericardium, there is an increased risk of stimulating the heart which leads to a drop-in blood pressure and a heart rate disorder.

For lung cancer surgery, surgeons should be aware of the concept of an absence of pericardium. Especially in thoracoscopic surgery, when separating the pericardial zone, if the boundary is not clear, the possibility of pericardial agenesis should be considered. Because of the absence of the pericardium, the upper left pulmonary vein and the left pulmonary artery are more easily dissected. Once the pericardium is found to be absent, attention should be paid to the protection of the heart, epicardium and the coronary artery during the operation.

The application of video-assisted thoracoscopic surgery (VATS) in the surgical treatment of lung cancer is becoming more and more common http://www.cgtvs.com/vats-leftpneumonectomy-with-pericardial-defect/?from=groupmes sage\&isappinstalled=1. However, Joon Suk Park and others have carried out a large sample report of conversion rates of $15 \%[11,12]$. There are main six causes for conversion. The first category was conversion associated with anthrax fibrosis of hilar lymph nodes, a common disease in Asian populations that causes lymph nodes to adhere to hilar structures (e.g., pulmonary vessels or bronchi). The second category was conversion due to a fused interlobar fissure, which complicates anatomic dissection of interlobar structures and hilar. The third category was conversion due to vascular (V) (usually PA) injury, the fourth category was conversion due to anatomic abnormality 


\section{Cancer Therapy \& Oncology International Journal}

of pulmonary structures, especially angeial structures. The Fifth category was conversion related to technical issues, such as stapler misfire or equipment failure. The sixth category was conversion related to arrhythmia [11-14].

Yet the VATS left Pneumonectomy with pericardial defect is not reported, and pericardial defects discovered intraoperatively, during the VATS left pneumonectomy, which is regarded as an indication of thoracotomy, are not reported. Although our patient had an abnormal heart rate and hypotension during the operation, we suspended operative interference and it was recovered within a few minutes. The patient's condition did not require a conversion to a thoracotomy.

It is reported that anesthetics on the surface of the pericardium can reduce cardiac stimulation. In this case, the patient's cardiac stimulation resolved itself and therefore, we don't know if a patient without a pericardium can anaesthetize the surface of the pericardium. There was also no arrhythmia and hypotension in our patient after the operation. Although arrhythmia and hypotension can easily occur under VATS left Pneumonectomy with pericardial defect, that is not a point of conversion to a thoracotomy. We think that there is no need for conversion to a thoracotomy if a pericardial defect is discovered during a VATS left Pneumonectomy. Therefore, the discovery of Pericardial defect during a VATS left pneumonectomy is not an indication of thoracotomy.

\section{References}

1. Galetta D, Spaggiari L (2015) Thymectomy and transpericardial nodal dissection. Thorac Cancer 6(3): 375-377.

2. Wang M, Chang TY, Yin WH, Yang YN (2016) Right Ventricle Mimics Right Atrium at First Glance: A Rare Case of Congenital Right Sided Partial Pericardial Defect. Acta Cardiologica Sinica 32(6): 748-750.

3. Verde F, Johnson PT, Jha S, Fishman EK, Zimmerman SL (2013) Congenital absence of the pericardium and its mimics. J Cardiovasc Comput Tomogr 7(1): 11-17.
4. Tomich J, Bhasin M, Philpott J (2013) The role of cardiac magnetic resonance imaging in the evaluation of congenital pericardial defects. Ann Thorac Surg 95(6): 2178-2180.

5. Abbas AE, Appleton CP, Liu PT, Sweeney JP (2005) Congenital absence of the pericardium: case presentation and review of literature. Int J Cardiol 98(1): 21-25.

6. Shimada Y, Yoshida J, Aokage K, Hishida T, Nishimura M, et al. (2011) Complete left-sided pericardial defect in a lung cancer patient undergoing pneumonectomy without closure of the defect. Ann Thorac Cardiovasc Surg 17(1): 67-70.

7. Jeong HJ, Seol SH, Seo GW, No TH, Seo MG, et al. (2014) Congenital partial defect of the left pericardium presenting as chest pain. Heart Lung Circ 23(12): e270-272.

8. Macaione F, Barison A, Pescetelli I, Pali F, Pizzino F et al. (2015) Quantitative criteria for the diagnosis of the congenital absence of pericardium by cardiac magnetic resonance. Eur J Radiol 85(3): 616624.

9. Chassaing S, Bensouda C, Bar O, Barbey C, Blanchard D (2010) A case of partial congenital absence of pericardium revealed by MRI. Circ Cardiovasc Imaging 3(5): 632-634.

10. Leschber G (2018) Video-assisted thoracic surgery: A global development. Chirurg 89(3): 185-190.

11. Kumar A, Asaf BB, Lingaraju VC, Yendamuri S, Pulle MV, et al. (2017) Thoracoscopic Decortication of Stage III Tuberculous Empyema Is Effective and Safe in Selected Cases. The Annals of thoracic surgery 104(5): 1688-1694.

12. Park JS, Kim HK, Choi YS, Kim J, Shim YM, et al. (2011) Unplanned conversion to thoracotomy during video-assisted thoracic surgery lobectomy does not compromise the surgical outcome. World J Surg 35(3): 590-595.

13. Samson P, Guitron J, Reed MF, Hanseman DJ, Starnes SL (2013) Predictors of conversion to thoracotomy for video-assisted thoracoscopic lobectomy: a retrospective analysis and the influence of computed tomography-based calcification assessment. J Thorac Cardiovasc Surg 145(6): 1512-1518.

14. Gazala S, Hunt I, Valji A, Stewart K, Bédard ER (2011) A method of assessing reasons for conversion during video-assisted thoracoscopic lobectomy. Interact Cardiovasc Thorac Surg 12(6): 962-964.

\section{Your next submission with Juniper Publishers will reach you the below assets}

- Quality Editorial service

- Swift Peer Review

- Reprints availability

- E-prints Service

- Manuscript Podcast for convenient understanding

- Global attainment for your research

- Manuscript accessibility in different formats

( Pdf, E-pub, Full Text, Audio)

- Unceasing customer service

Track the below URL for one-step submission https://juniperpublishers.com/online-submission.php 